\title{
The Research on Construction Function of Critical Thinking
}

\author{
Gao Aihua \\ School of Continuing Education, Yanshan University, Qinhuangdao, China
}

\section{Email address:}

gaoaihua@ysu.edu.cn

\section{To cite this article:}

Gao Aihua. The Research on Construction Function of Critical Thinking. International Journal of Philosophy. Vol. 5, No. 1, 2017, pp. 1-6. doi: $10.11648 /$ j.ijp.20170501.11

Received: January 11, 2017; Accepted: January 25, 2017; Published: March 1, 2017

\begin{abstract}
This paper has long been misunderstood for some critical thinking exist, such as critical thinking is negative and destructive problems such as the evolution from the essence and the development of critical thinking to define the trajectory; critical thinking in creative work, the role of construction and social cultural functions in the perspective of critical thinking, discusses the construction of function. The purpose is to promote the comprehensive and accurate understanding of critical thinking, to promote the development of critical thinking education in our country, and to play the role of critical thinking.
\end{abstract}

Keywords: Critical Thinking, Creativity, Construction Function

\section{Introduction}

At present, many students in our school are not fully aware of the nature of critical thinking, and there are still some misunderstandings. For critical thinking, many people only pay attention to the literal meaning of the two words of criticism, that critical thinking is negative, exclusive, destructive, at least, critical thinking is a negative. In fact, critical thinking as a way of thinking is neutral and objective, using critical thinking is clear the significance of learning objects in the learning process, the learner's own value, position and framework to show, is the construction process of scientific theory. Lack of "critical thinking" can lead to rigid thinking, the academic community is also a lack of real academic exchanges and dialogue, in the daily life, work in the field not be innovative, achievements. Therefore, a comprehensive and accurate understanding of the essence and function of critical thinking is very necessary for the development of critical thinking teaching in china.

\section{On the Construction Function of Critical Thinking}

At present, the definition of critical thinking has not yet formed a unified. Because of the scholars from different direction and different disciplines to define critical thinking, so there are many types of the definition of critical thinking, and present different development status in different periods.
The definition of critical thinking, academic circles have not yet reached a consensus of the integration of it remains to be further efforts to Google. Development of the definition of critical thinking has experienced four stages: from 1910 to 1910 for the first stage. Mainly based on the scientific method of Dewey advocates "reflective thinking" to start, think carefully about any belief, knowledge, looking for reason to support it and then to conclusions; From 1940 to 1961 for the second stage, it to glazer, representative, Russell Smith and thoughts on the basis of acceptance of Dewey views to develop, the "state examination" included. From 1962 to 1979 as the third stage, representative to the meaning of critical thinking, narrow, only contains statements, exclude problem solving and scientific method. He expressed as: critical thinking is "to decide what to believe or do reasonable, reflective thinking"; For the fourth stage, from 1980 to 1980 in earnest, can peck, Siegel and Paul's definition, critical thinking has been widening, includes all aspects of problem solving. Habermas equating critical thinking to "liberation" learning ", teach people to learn from my insight into new trend, dominate their life and work of the external force. [1]

From the point of formation of the definition of critical thinking, critical thinking question boldly Qi Dewey is, cautious assertions. Of existing knowledge, theory and belief before accepting it, to be serious and careful reflection and inquiry. Dewey defined critical thinking is the essence of the objective, neutral, neither for nor against it, don't blindly make a decision - delaying judgment. 
At present, the definition of critical thinking is used more Ennis's view: reasonable, reflective thinking, its aim is to decide our beliefs and actions. Ennis thinks, the public usually think that critical thinking is negative, doubt, once you have the evidence of a certain point, reason, critical thinking will no longer be skeptical. [2] in fact, even at exposing mistakes and shortcomings in critical thinking as the goal of thinking, if it has the basis of an objective and fair, whether it is not only qualitative, it is constructive and is in no wrong judgment on the basis of the construction.

With the evolution of the concept of critical thinking, the construction of the it function is becoming more and more prominent. The concept of critical thinking is about things, careful observation, analysis, judgement, determine what should believe, should not believe what, and to evaluate its, determine its correct or not, is to deal with. Recent critical thinking, though still want to judgment theory, hypothesis, argument, but it is to construct new to replace the original. Therefore, critical thinking is more and more is considered to be structural or constructive. Under this background, researchers think: many critical thinking, views on the existing conclusion, should first take look for trouble, to reveal the drawback of the intention to analyze and solve problems, and then construct the correct these to these defects.

This goal to reveal inadequate approach, is considered by some critical thinking researchers is a good way to construct. Popper thinks, tried and proven scientific theory might be wrong, better theory can only be produced by correct its mistakes. So, through the strict examination and critique way to explore the to go wrong, it is the practice of the development in the first place, is a more effective way to promote the development of knowledge. [3] promote the development of things and the generation of new theory is the negation of old affairs on the basis of the unreasonable factors, therefore, find errors not hinder people's understanding, but promote the generation of new theory, new things. So, here's the drawback of the method is to construct better.

From the definition and development of critical thinking, critical thinking is to let people learn to think in a various skills, to build the correct, scientific and rational belief, theory, rather than to simply deny others, and others. In fact, the main purpose of the critical thinking is to promote people's understanding of things more, deeper, ask people in such aspects as scientific, cultural knowledge, the growth of the value, critical thinking is the most effective tool for this purpose. For critical thinking education, some people only understand "criticism", rather than building. Due to the misunderstanding of the political, to some extent, affected the critical thinking education in our country widely.

In addition to the logical function of argumentation, critical thinking is a kind of learning and research skills, attitudes and habits. Critical thinking can integrate all kinds of skills and use it effectively, and enhance the ability of thinking in the study and daily life. Research methods of Chinese traditional Confucian classics, namely in the "book of Rites" in the doctrine outlined: erudite, questioning, deliberative, discernment, Dusing, known as the "five step", which contains critical thinking in this area, we can extract the thinking the corresponding content from the critical functions such as critical thinking "insight, analysis, inspection, evaluation and reconstruction etc.. To learn about the traditional "five step", if you put aside the containing "goodness" and "truth" to the self-cultivation of the traditional values and other content, is equivalent to the critical thinking, thinking, thinking to ask. The emphasis of the deliberative is cautious and careful thinking, confirmation, critical thinking is not a simple negation, it is necessary to think, it is prudent to identify the authenticity, seeking truth, make about what to believe or do what the "rationality" of decision. Critical thinking is the existing knowledge, expression and problem-solving methods, strategies of questioning, denial, the theoretical system of knowledge, such as monitoring and repeated thinking, evaluation, demonstration. The cultivation of critical thinking is established on the basis of that, a lot of the collection of information, based on certain facts, based on the theory of objective judgment, fully thinking, analysis of the facts and evidence, and then make a decision. Each step of the "five steps" embodies the characteristics of critical thinking. "Learned" refers to the knowledge of critical thinking in before judgment is also required to have large and rich information; "questioning, deliberative, discernment" refers to the process of reflection, critical thinking, identify the question. "Dusing and critical thinking in common, after learning, thinking of the link to guide our behavior. But they also have differences, that is the critical thinking of requirements analysis, evaluation and demonstration of construction to withstand the test of logic analysis, strict derivation, and the "five step" is careful, careful thinking, confirmation. Critical thinking focuses on the study of knowledge and the pursuit of truth. While learning the five step in the acquisition of knowledge at the same time, pay attention to people's moral cultivation.

\section{The Construction of Critical Thinking Is Also Reflected in the Creative}

First of all, on the side of the implementation process of critical thinking, critical thinking can build a new scientific theory. People usually speak no break no stand, under the scientific evaluation and verification of critical thinking, and often wrong, does not accord with objective conclusion is negative, then, the correct, new scientific theory was born. From this point, the construction of critical thinking feature also reflect in creative ways.

People to implement the results of the critical thinking includes two aspects: one is the theory of knowledge, has been formed if after strict inspection, verification, can still stand, then we should be sure it. The second is completely opposite to the former results, then overthrow, broke through the predecessors' point of view, and thus a new theory. 
Critical thinking advocated by scientific skepticism to treat existing knowledge and judgment of authority, no criticism of the old ideas and things, there is no innovation. The construction of any scientific theory, all need through the thinking of the structure, negative and critical. If there is no critical thinking, the existing theory, ideas won't get a new development. The construction of a new theory, is often in Critically reflect on the premise of both the concept, process and conclusion on the basis of, through analysis, synthesis, judgment, reasoning, generalization, abstraction, and a series of measures.

Secondly, from the perspective of the forming process of creative thinking, critical thinking. a British psychologist Australia (G.W) is put forward, the creative thinking process contains four stages, namely the preparatory stage, run-up, anacreontic and verification phase. [4]

The process of the first phase of the reserve a lot of information, data and practical experience, the identification, handling, for these things need to use critical thinking. Critical thinking can be to deal with a lot of basic information, in the process of the main work of critical thinking is how to deal with all kinds of information is more scientific and reasonable. Doubt is the starting point of innovation, it is the necessary premise of creative thinking, promoting a healthy and critical thinking. About things healthy skepticism, helps people to the discovery of science, creative thinking is a kind of break the routine, dare to transcend, imagination thinking activity, it can be incorporated into a variety of ideas. And critical thinking is deliberating, selective, eliminating the possibility of irrational, in-depth and careful observation, the relationship established between things, to distinguish the differences between exploratory questions, guiding people actively to think rationally in the existing theoretical knowledge, to build scientific and correct point of view. Without the use of critical thinking, this part of work was unable to complete.

In the second, three stages of the creative thinking, creative thinking of brewing, clear stage, also is a new theory, new ideas of thinking, and puts forward phase, is also inseparable from the critical thinking. Two stages of this work is to work in the previous step, on the basis of careful research institute to provide information, assumptions and hypothetical solution is put forward. At this stage, need some of the critical thinking of the thinking methods, such as questioning of the judgment of the original thing, such as breaking mind-set, including authority, conformity, experience and so on box; Extended thinking from the perspective of to think the same things from various angles, such as critical thinking scholar hunt (David Hunter) has put forward "three of" principle, for breakthrough thinking framework of the individual, found the multidimensional nature of the object: for a problem, should look for three different perspective; On a target, and find out three kinds of action; Define an idea, and find out three examples; Searching for an argument, three counters. At this stage of the formation of the theoretical questions, identify its authenticity and comparing, gradually new and feasible scheme is put forward. In addition, the two stages of the use of some creative thinking methods, such as inspiration, intuition, fantasy, also is in the supervision and restriction of critical thinking. Such as is of great significance to the innovation of the imagination of thinking ability, critical thinking while pay attention to the rigorous, rational attitude and strict reasoning, proof ability, but it does not hinder people's imagination, it can supervise the imagination of the people, make it more scientific and reasonable to people's production and life, even, critical thinking also provide favorable conditions for people's imagination.

The last stage of the creative thinking, it is proof of these schemes are proposed by reasoning, and a series of evaluation, testing work. If is good at creating new ideas, new ideas, innovative thinking, critical thinking, good at make evaluation to view, thoughts, aims to develop new ideas, perfecting the existing views. Evaluation and assessment is an important content of critical thinking, critical thinking is based on rigorous evaluation of evidence and examples, through strict reasoning, is a kind of evaluation method based on logic and evidence. Inspection for innovation is a complex process of critical thinking, we must according to the different characteristics of the concrete scheme to solve the problem put forward the corresponding strategy. People can use critical thinking methods to test, first check the premise of a new conclusion is real, fully, can the premise condition must be new conclusions, verify the invented new method is scientific and reasonable, is in line with the new achievements or the extent to which conform to the practice, and applied in practice. Experiments show that data to support the new results can reach how much, and so on. After assessment, inspection, may have some new ideas, there may be some parts was established, need further correction, or even some view completely wrong, need to overthrow, to ask questions. Not only focus on critical thinking, more positive affirmations to multi-angle to assess the argument, and reflective analysis and investigation. After a long period of practice has proved that some new theory and new understanding of the strict verification only through critical thinking, to become a real innovation.

Because creative thinking is not pay attention to every step of the right and wrong, and critical thinking not only insisted on each link in the process of thinking is correct, it also evaluates some examples or view is valid. Therefore, in the process of creative activities without critical thinking. Logic is indispensable to any subject for research, innovation is an important part. In informal logic deduction, induction and analogy reasoning, deductive methods such as hypothesis is the basic means of innovation activities, and critical thinking and the formal logic on the above content is basically the same. Therefore, the stronger the one critical thinking ability, the innovation ability is stronger.

The theory of argumentation in critical thinking reveals the structure of argument, gives the standard of evaluation, and the principles and methods of constructing argumentation. Include: 1, to identify the structure of the argument, to find arguments, arguments, arguments and 
those who violate the clear statement but in the debate is essential to the underlying assumptions and assumptions. 2, evaluate the rationality and validity of the existing arguments. Including some key concepts and topics can be accurate and clear; the premise, in the demonstration of the implicit premise of authenticity and acceptability; check the precondition and the conclusion of contact is associated with logic, semantics or mind; the argument to support the argument and so on. 3 , to create a new, more scientific and reasonable argument, the establishment of a more reasonable new argument. The traditional China Mingbian in science, including proof of rebuttal, is about reasoning knowledge. The argument is the collision of two kinds of positive and negative views, is contradictory thinking of confrontation, is the use of critical thinking where the most concentrated, the most typical and most widely, it will be the case in the establishment of other ideas and motivations of suspicion and judgment. Analysis of Mingbian analysis and reasoning method by focusing on the concept, the reasoning method is mainly named science thinking method based on "class" based on the concept of class and push aid, namely "reasoning". It is in accordance with the two different things and phenomena in the "class" or "type" attribute meaning with the same or similar in order to ensure the validity of the argument, debate tool. The Mohist School comprehensively expound the concept, judgment and reasoning theory, a unique DAC, push, check and inference method. "Mojing" essence, study the principle and way of reasoning. From the point of view of the argument is: on the demonstration method used in the process of argumentation, is to obtain the correct understanding and use of knowledge or reasoning, embodies the nature of reasoning mode. In critical thinking, reasoning forms in accordance with the demonstration of the different argument can be divided into three basic types, namely deductive reasoning, inductive reasoning and plausible argument.. Is a plausible argument in the case of incomplete information reasoning, it includes analogical reasoning. "Mojing" lists a variety of belonging to the analogy, inductive and deductive reasoning, we first look at the analogical reasoning, it is prerequisite to have some similar properties from the two things, the conclusion derived other attributes of the two things of the same reasoning, which is composed of individual of individual reasoning.

1, "monarch". "For also, for him and also in Ming dynasty. "That is to say something like this to illustrate this. For example, "said the" first "also considered to have the requirements, and shall not, if the wind." 2, "Beijing opera". Mou also, but also for all than speech. "Cancer is the use of two phase, equal or the same meaning" speech "to prove the analogy. Such as "small" in the "white horse, Ma also; by white horse, riding a horse also. "3," aid". "Aid, said: 'my son however, Xi alone may not be so? 'help' is to cite the other argument to prove their similarity. 4, "push" push "? Also, which is not taken, as the person, to the. "That is not in favor of the argument that the other party, and the other is in favor of the argument, belong to the same, and confute. "Mojing" using the analogous argument, implicit inference deductive and inductive. "Push in Mojing", including a variety of inference based on the similar relationship, both from individual to individual with the characteristics of analogical reasoning, but also from individual to general and inductive reasoning, deductive reasoning from the general to the individual. In order to improve the validity of inductive inference, it has also taken some effective measures: "by the said" said: "for it, this thought so. "Is a list of individual positive cases, to draw a general proposition. "Mojing" thought this kind of reasoning to make hasty generalization error, need to use "check", paying attention to the discovery and reference to the limit and correction of counterexamples. The nature and theory about reasoning Mohist presented late in the classic mode is also a lot of deductive reasoning. For example, we are more familiar with the "three things" reasoning model, is a typical representative of the deductive reasoning model. "Three things, then words enough to live". Enough is enough, enough meaning, which itself contains a certain meaning. This is a sufficient condition for the hypothetical proposition: if $\mathrm{P}$ then $\mathrm{q}, \mathrm{p} \mathrm{q}$, its characteristic is, certainly before $\mathrm{P}$, after $\mathrm{Q}$ will certainly, logical relationship between the antecedent and the consequent inevitable that this proposition is so deductive nature proposition. Furthermore, a "Mojing" in the "effect" type inference problem. There are many scholars on the "effect" type inference understanding, some of the "effect" as a topic for evaluation is not standard, some of the "effect" to establish the formula into the formula or that it is a copy, but they think the same point is: "effect" is a kind of deductive logic, and only with the push of "effect" is correct. [3] the main content from the demonstration to discuss the similarities of Mohism and critical thinking theory, the color is very bright, remarkable features of critical thinking. The main function of the fallacy theory in critical thinking is to ensure the smooth progress of the argument.

\section{The Construction Function of Critical Thinking in Social Culture}

At present, the implementation of the critical thinking teaching, research, is as a research direction of logic, and a specific rational thinking training course. Logic as a course of study thinking, it is the foundation of all other discipline construction. The basic concept of these disciplines, the determination of category, system and so on are the logical basic principles. Logic as a basic subject role, has been in the 1970 s by the United Nations educational, scientific and cultural organization confirm, at present, it has developed into a number, reason, and tied for the basic subjects of heaven, earth, was quoted. From logic as the basis of different subjects and scientific research the Angle of the tool, you can be sure logic functions in the scientific research in the field of construction. At the same time, the logic rationality of the humanistic spirit of today's society culture 
construction function gradually known by people and excavation.

Therefore, critical thinking and logic have close relations, people in the study, using the basic concepts, techniques and methods, its philosophy thought and rational spirit of modern society's important construction effects also has attracted widespread attention. [5]

Critical thinking in give people a technique to study the principle, method, at the same time, also cultivate the people a kind of attitude, spirit and temperament. Although had many different critical thinking in the history of the researchers, but had the same ideas, and there's nothing we can summary the common part here. From Ennis, Paul, put forward primary three aspects, the spirit of critical thinking is the truth, justice, and reflect on, and has stipulated some reason for this specification, such as intellectual honesty, comprehensive, open and clear, careful, cautious, introspection, makes every effort to understand and believe that the rational reflection. This is an ideal critical thinking should have the psychological culture of quality, is also a modern qualified citizens shall have the connotation. Contemporary people in making decisions, needs to be critically listening, reading, watching, speech, writing, in order to make a more scientific and reasonable decision, maximize access to more favorable for their survival and development opportunities. If a person often use critical thinking, can greatly improve their thinking ability, can promote the progress of the study, work; If often use critical thinking and creative thinking can make innovation in practice, to become creative talents. If many people do this, our country will build a creative country.

Critical thinking helps us to build a socialist harmonious society, harmonious society is not advocated everyone's opinion as uniform as possible, Ting $\mathrm{Bu}$ De differences and opinions, more than ever in the history of the arbitrary and blind. Critical thinking that people at the existing theory, views on social health, carefully thinking and analysis. Democracy, science, and the rule of law society is that we need to build a harmonious society today, the formation and operation of modern society depends on the rational ability of citizens to support, democracy requires citizens have an open mind temperament of critical thinking, critical thinking, including interpretation, analysis, evaluation, inference, explanation, self calibration, it can improve the quality of citizens, the basis of individual autonomy. Need to demonstrate need to demonstrate at the same time, democracy, science, rule of law need to demonstrate that the argument is the main content of critical thinking, it is actually thinking of informal reasoning as the research object and teaching content. Respect argument is to advocate science and scientific spirit, to establish a real constitutional democracy requires critical thinking and critical thinking includes not only the skills of dimensions, including dimensions of temperament and spirit. The practical characteristic of harmonious society characterized by consensus to achieve and maintain and update, but the consensus is based on the main body of each other can be recognized on the basis of critique the effectiveness of the inspection requirements.

Critical thinking is an open spirit under the guidance of dialectical reason of cognitive thinking, communication, dialectic, open approach is critical thinking guiding principle of education. Under the background of the world, we also cannot leave the critical thinking. Today's world is an open world, a variety of ideology, the era of multicultural coexistence, no matter in such aspects as ideology, academic, it can give people enough breadth of thinking. We want to be in critical thinking and open spirit and the guidance of dialectical reason from understanding the world. Critical thinking advocated fair and objective judgment should be established on the basis of a certain openness, universality, usually people think that the range of choice in decisions is wider, more reasonable. Thus, in this open any of the countries in the world advocate such values and quality education are necessary.

\section{Conclusion}

In a word, critical thinking is not purely negative, destructive, more constructive. In sexual issues, give full play to the critical thinking construction to develop critical thinking education is an effective measure. We according to the level of demand for critical thinking is different in population of different critical thinking education of different levels, this is an effective way to cultivate citizens' critical thinking level in our country. About critical thinking teaching, first of all, we should set up the correct teaching view, want to let more students to correct understanding and the understanding of the concept of critical thinking, the basic theoretical knowledge such as trajectory, in practice, however, also requires students to master and use its methods, skills, and learn about critical thinking the philosophy thought and rational spirit. That is to say, not only requires the educators to master the skills of critical thinking, method, should advocate people more methods and skills of critical thinking in practice consciously use, form a good habit, internalizing a kind of spirit and temperament. Critical thinking on the development of our society, science, culture plays an important role in construction, therefore, extensive and in-depth critical thinking teaching is of great significance in our country, should cause enough attention to relevant departments.

\section{Acknowledgements}

Thanks GJXH 2013 - 43 Higher Education Research Institute of Hefei Academy. Name of the project: the critical thinking course and the cultivation of college students' innovative ability in Colleges and universities of Hebei.

\section{References}

[1] Wu Hongzhi. Definition and consensus of multi perspective of critical thinking $[\mathrm{J}]$. Journal of Yan'an University (SOCIAL SCIENCE EDITION). 2012, 2. (5-6). 
[2] Wu Hongzhi Liu Chunjie. Critical thinking [M]. Xi'an: Shaanxi people's publishing house. 2005.6. (3-4).

[3] Xiao Xing. Research on the cultivation and training of innovative thinking $[\mathrm{M}]$. Nanchang: Jiangxi University Press. 2008.1.

[4] Liu Yetao. The social and cultural function of critical thinking [J]. academic forum. 2009.9. (32-33).

[5] Anderson, T. 2005. Critical Reading Skills Activities for High School. [Z].

[6] http://www.ehow.com/way_5730199-critical-skills-activitiesschool.html.

[7] Applegate. \& M. Dekonty. 2006. Using the Critical Reading Inventory to Guide.

[8] Differentiated Instruction [C]. College Reading Association Yearbook (28).

[9] Bartlett, F. C. 1932. Remembering [M]. Cambridge University Press.
[10] Liu wei, Guo hai-yun. (2006). Critical reading teaching model experimental study [J]. Foreign language community, (3): 14 to 18 .

[11] Sue full-color, (2008). The theory of critical thinking and the English reading teaching [D], guang xi university.

[12] Sue full-color, pro. (2012). The status analysis of critical English reading teaching in China [J]. Journal of guangxi normal university: philosophy Social science edition, 33 (3): $115-118$.

[13] Li tai-ping, Liu Yan-nan. Education research, from theory to practice a rational and rational education theory Relation to education practice. Education research, 2014, 4-10.

[14] Qi dong-fang, Hou huai-yin. Retrospect and prospect of Chinese higher education philosophy research. Journal of hebei university (philosophy Learning science edition), 2014, 77-82. 